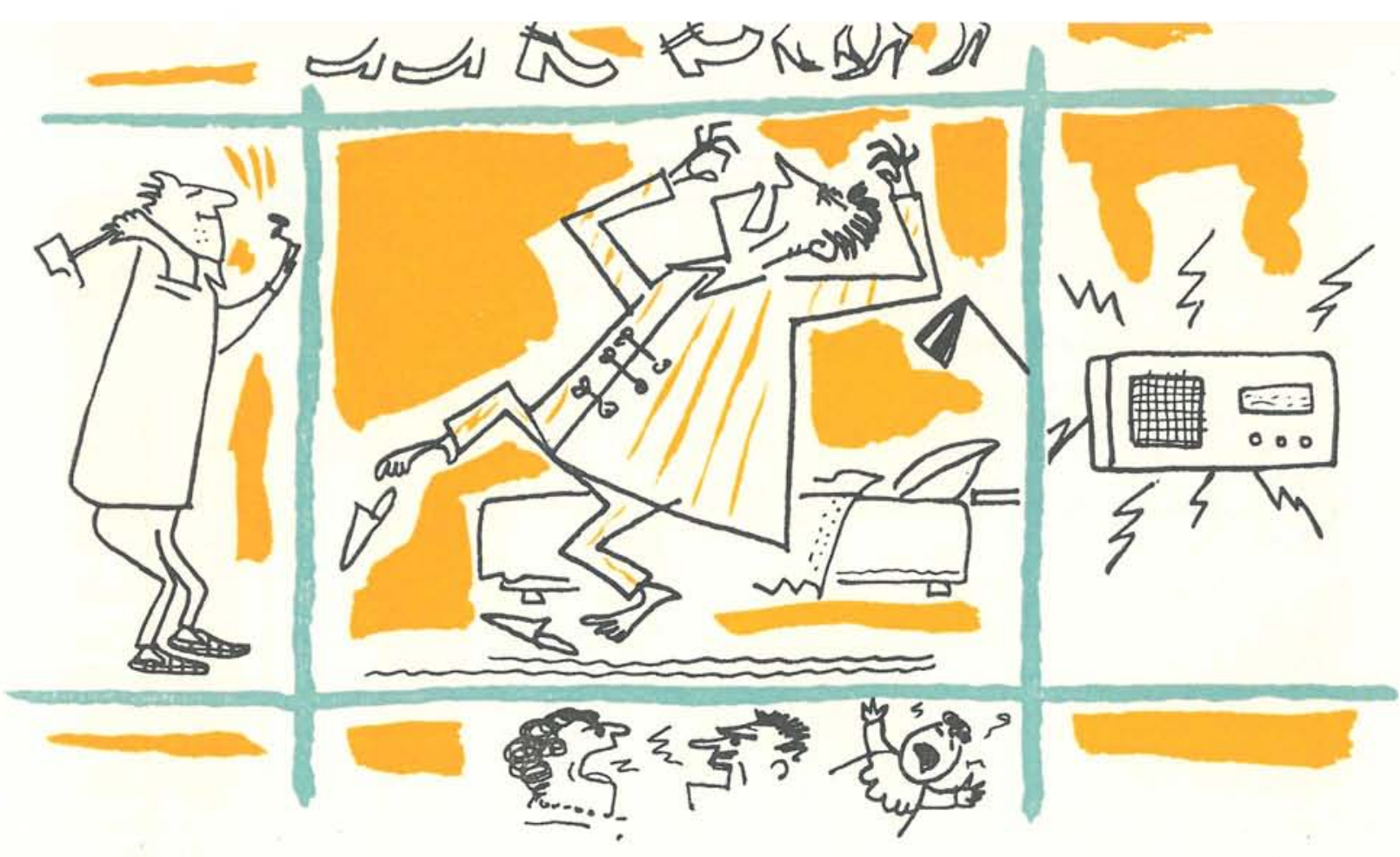

\title{
el ruido en instalaciones de aire acondicionado
}

\author{
A. ALAMAN, ingeniero industrial \\ Jefe de la División de Instalaciones del I. E. T. c. c.
}

$340 \cdot 4$

\section{sinumpois}

Se expone en este artículo una idea general del problema del ruido en instalaciones de aire acondicionado, problema que preocupa hondamente en los medios técnicos correspondientes, por su gran influencia en el confort de los edificios.

Se dan las cifras de niveles de presión sonora admisibles, recomendables para un Pliego de Condiciones, y el procedimiento para comprobar si las instalaciones terminadas lo cumplen, así como los medios normalmente utilizados para evitar la emisión
de ruido a los locales habitados.

Para el estudio del confort sonoro se recomienda tener en cuenta, tanto en el Pliego de Condiciones (mediante el empleo de la escala "Ax) como en el estudio de la eliminación de ruidos, al proyectar la instalación (mediante las curvas espectrales que cada nivel sonoro, con los que es posible lograr, sino, de acuerdo con la tendencia mundial, el espectro de frecuencias de tista le será de gran ayuda contar con la necesaria cooperación de los fabricantes los cuales facilitarán datos referentes a las propiedades acústicas de sus productos, tanto de los que puedan ser emisores de ruido como de los que vayan a ser utilizados como absorbentes o aislantes.

La mecanización de nuestras ciudades y casas ha traído consigo un aumento de ruido en el interior de nuestras viviendas y oficinas, ruido que, en orden al mayor confort, se está estudiando y regularizando para evitar que sobrepase unos límites adecuados.

Una de las fuentes ruidosas pueden ser las instalaciones de aire acondicionado. Sin embargo, aunque este específico problema no está totalmente resuelto, existen soluciones con las que, con un estudio cuidadoso, es posible conseguir condiciones de confort en las salas o habitaciones ocupadas.

A continuación se indican, a grandes rasgos, el planteamiento del problema y algunas de las soluciones más utilizadas en instalaciones de aire acondicionado, para evitar, o al menos atenuar, la emisión y propagación del ruido a los locales habiados. 
VALORES GENERALES DE NIVEL SONORO DE INSTALACIONES DE AIRE ACONDICIONADO, recomendados por The American Society of Heating, Refrigerating and Air Conditioning Engineers

$$
\text { TIPO DE LOCAL }
$$

$\underset{\text { Máximo }}{\text { Nivel sonoro A, en db }} \underset{\text { Medio }}{\text { Mínimo }}$
Máximo C. nivel, en db

AUDITORIOS Y SALAS DE MÚSICA:

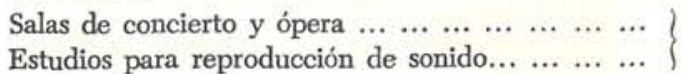

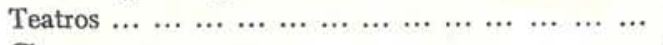

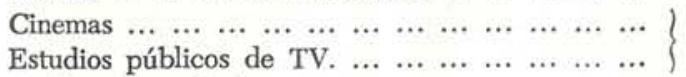

Pasillos y vestíbulos

IGLESIAS Y ESCUELAS:

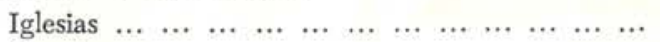

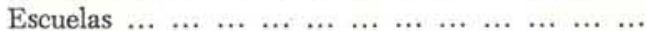

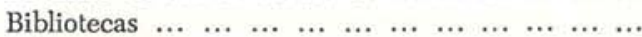

Laboratorios $\ldots \ldots \ldots \ldots \ldots \ldots \ldots \ldots$





HOSPITALES Y CLINICAS :

Habitaciones privadas $\ldots \ldots \ldots \ldots \ldots \ldots$

$\left.\begin{array}{llllllllllll}\text { Quirófanos } & \ldots & \ldots & \ldots & \ldots & \ldots & \ldots & \ldots & \ldots & \ldots & \ldots & \ldots \\ \text { Salas generales } & \ldots & \ldots & \ldots & \ldots & \ldots & \ldots & \ldots & \ldots & \ldots & \ldots\end{array}\right\}$

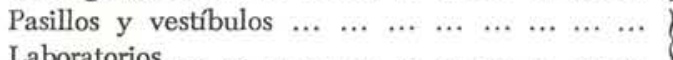

$\begin{array}{llllllllllll}\text { Laboratorios } & \ldots & \ldots & \ldots & \ldots & \ldots & \ldots & \ldots & \ldots & \ldots & \ldots & \ldots\end{array}$

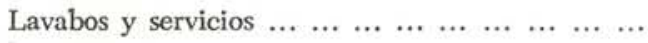

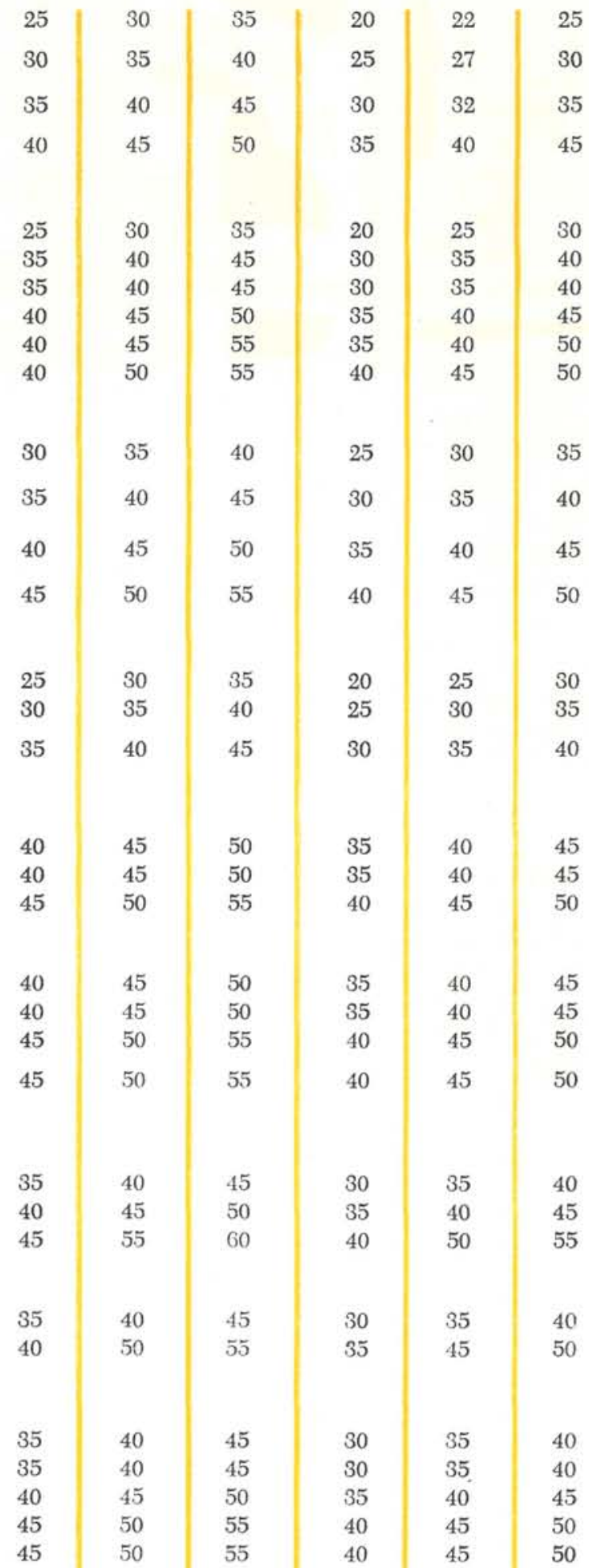

RESIDENCIAS :

Hoteles privados en el campo... ... ... .........

Hoteles privados en la ciudad... ... . . . . . . . . .

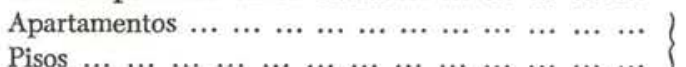

RESTAURANTES Y CAFETERIAS:

Restaurantes $\ldots \ldots \ldots \ldots \ldots \ldots$

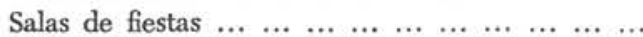

TIENDAS Y ALMACENES:



Grandes almacenes (plantas superiores) $\ldots \ldots \ldots$

Grandes almacenes (planta principal)... ... ......

Pequeñas tiendas $\quad \begin{array}{lllllllllll} & \ldots & \ldots & \ldots & \ldots & \ldots & \ldots & \ldots & \ldots & \ldots\end{array}$

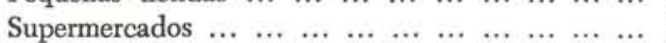

SALAS DEPORTIVAS:

Palacio de Deportes ... $\ldots \ldots \ldots \ldots \ldots \ldots \ldots \ldots$

Boleras y gimnasios $\ldots \ldots \ldots \ldots \ldots \ldots \ldots \ldots$

Piscinas cubiertas $\ldots \ldots \ldots \ldots \ldots \ldots \ldots \ldots \ldots$

ESTACIONES DE VIAJEROS:

Sala de billetes $\ldots \ldots \ldots \ldots \ldots \ldots \ldots \ldots \ldots$

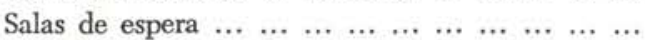

HOTELES :

Habitaciones individuales $\mathrm{y}$ «suites» $\ldots \ldots \ldots \ldots$

Salas de baile $\mathrm{y}$ banquetes $\ldots \ldots \ldots \ldots \ldots \ldots \ldots$

Pasillos y vestíbulos $\ldots \ldots \ldots \ldots \ldots \ldots \ldots$

$\begin{array}{lllllllllllllll}\text { Garajes } & \ldots & \ldots & \ldots & \ldots & \ldots & \ldots & \ldots & \ldots & \ldots & \ldots & \ldots & \ldots\end{array}$

Cocinas y lavaderos $\ldots \ldots \ldots \ldots \ldots \ldots \ldots$

35




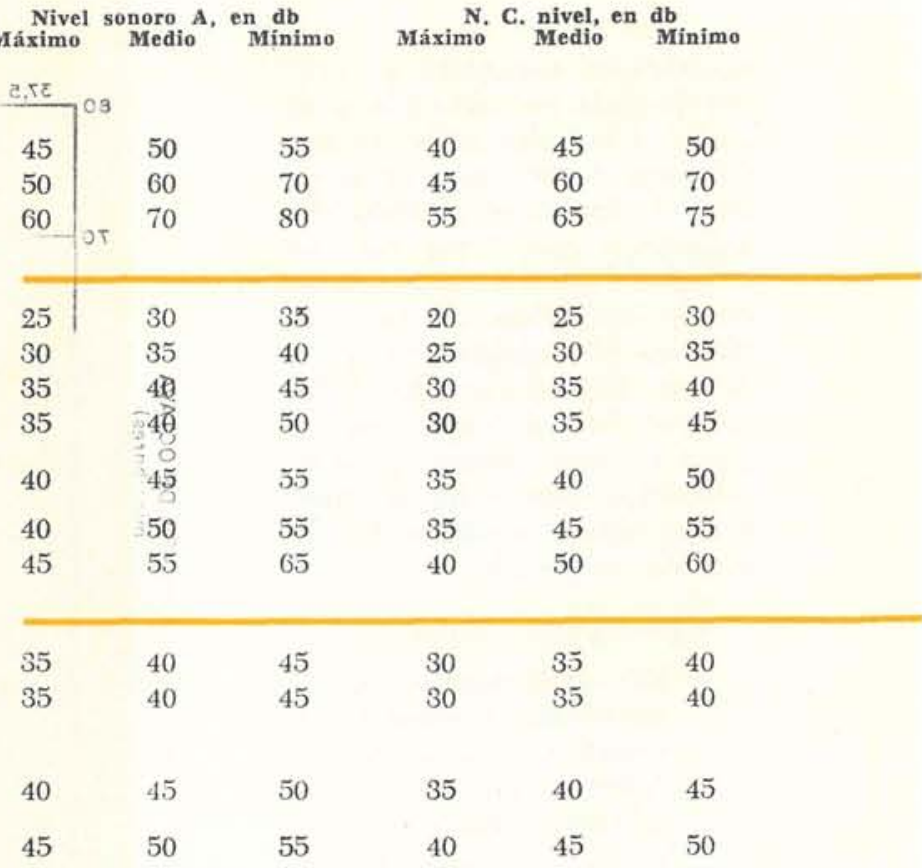

\section{FABRICAS :}

Oficina del encargado o capataz ... ............

Salas de montaje y maquinaria ligera ... ... . . . .

Fundiciones y maquinaria pesada... ... . . . . . ...

\section{OFICINAS:}

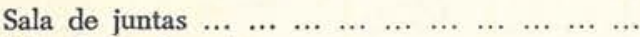

Sala de conferencias $\ldots \ldots \ldots \ldots \ldots \ldots \ldots \ldots$

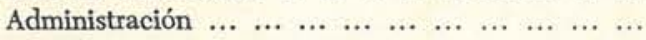

Salas de comprobación y recepción $\ldots \ldots \ldots \ldots$



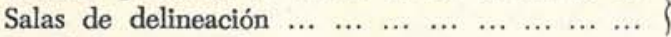



Salas de tabulación y computación $\ldots \ldots \ldots \ldots$

EDIFICIOS PUBLICOS:

$\begin{array}{lllllllllllll} & \text { Bibliotecas } & \ldots & \ldots & \ldots & \ldots & \ldots & \ldots & \ldots & \ldots & \ldots & \ldots & \ldots\end{array}$

Museos y salas de justicia ... ... ... ... ... . . .

Correos $\ldots \ldots \ldots \ldots \ldots \ldots \ldots \ldots \ldots \ldots \ldots \ldots$

Salas generales en bancos $\ldots \ldots \ldots \ldots \ldots \ldots$

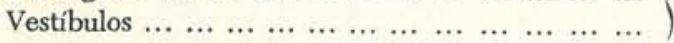

Lavabos y servicios $\ldots \ldots \ldots \ldots \ldots \ldots \ldots$



Las condiciones de confort exigen unos límites máximos de ruido. Estos límites son distintos para diferentes habitaciones o edificaciones, según el destino que éstas tengan, bien por el tiempo de permanencia normal en ellas, bien porque la actividad que se va a desarrollar en las mismas requiere un silencio mayor o menor, siendo, por- Łarito, distinto el efecto perturbador del ruido en unas y otras. Un restaurante no debe reunir las mismas características sonoras que una vivienda, una escuela, un hospital o una sala de conciertos.

Los valores máximos de nivel sonoro admisible en cada tipo de habitación están especificados en diversas normas existentes, de acuerdo con la influencia que en el hombre ejercen las distintas clases de sonidos en diversos ambientes.

El efecto que un ruido produce en el oído humano es función de la intensidad y de la frecuencia del mismo. Para una misma intensidad, por ejemplo, un sonido de baja frecuencia puede ser perfectamente admisible, mientras que otro de alta frecuencia puede llegar a producir incluso una sensación dolorosa. De ahí la necesidad de que al dar una cifra límite del nivel sonoro admisible esta cifra por sí sola tenga en cuenta la intensidad máxima admisible en cada frecuencia de acuerdo con el efecto fisiológico de la misma.

Una ponderación de los distintos tipos de sonido se obtiene utilizando la «escala A» de nivel de ruido, usada normalmente para evaluar el sonido en locales habitados. La apreciación del nivel de ruido en escala A se hace fácilmente por lectura directa en aparatos sencillos y comerciales, que automáticamente tienen en cuenta la distinta sensibilidad del oído humano a las distintas frecuencias. Para un Pliego de Condiciones son recomendables, por su adaptabilidad al confort y por la facilidad de comprobación en instalaciones realizadas, los valores de la escala A de la tabla adjunta.



La evaluación del nivel sonoro, ponderando la intensidad admisible en las distintas frecuencias, permite una selección más adecuada en la eliminación de los ruidos en un acondicionamiento de aire.

El espectro sonoro de cada una de las máquinas o dispositivos utilizados en acondicionamiento de aire es distinto entre unos y otros, y con frecuencia muchos de ellos emiten sonidos puros (acompañados o no de sus armónicos) o, al menos, agrupados en torno a una frecuencia determinada. Análogamente, el espectro de absorción acústica de los elementos, materiales o dispositivos aislantes o 
absorbentes utilizados es propio de cada uno de ellos, y su poder eliminador suele ser mayor para ciertas frecuencias se gún el tipo $y$ el modelo del aislante o absorbente de que se trate. Todo ello aconseja un estudio cuidadoso de las condiciones de emisión de ruido y de los dispositivos silenciadores que han de colocarse, así como el lugar óptimo para su colocación, con el fin de obtener el mayor rendimiento del material empleado.

Este estudio requiere:

- El conocimiento de las intensidades sonoras admisibles para cada frecuencia, en cada nivel máximo sonoro.

- El conocimiento del espectro sonoro emitido por cada aparato.

- El conocimiento del comportamiento como absorbentes o aislantes acústicos de los materiales o dispositivos usados con este fin en las instalaciones de aire acondicionado.



\section{a) Presión sonora máxima admisible para distintas frecuencias.}

Para cálculos de proyecto, la presión sonora máxima recomendada para cada frecuencia, de acuerdo con el nivel de ruidos admisible para una habitación (véase tabla), viene dada en el gráfico de la figura 1.

En este gráfico, las líneas llenas corresponden a las máximas presiones sonoras admisibles atendiendo a las condiciones normales de confort (los diversos criterios de nivel sonoro-NC-vienen indicados sobre las líneas). Sin embargo, en habitaciones en donde la sensación de confort es menos exigente que la necesidad de una buena apreciación de la comunicación hablada (por ejemplo, en salas de conferencias, en donde el confort tiene menos exigencias que en una oficina, por estar las personas menos tiempo en aquélla, pero en donde, en cambio, se precisa una ausencia de interferencias sonoras con la conversación), pueden admitirse mayores intensidades sonoras en bajas frecuencias, utilizándose en estos casos, como criterio de proyecto, las líneas de trazos de la figura 1.

\section{b) Fuentes de ruido.}

Raros son los elementos componentes de una instalación de aire acondicionado que no producen ruido, si bien, afortunadamente, el evitar que este ruido sea transmitido a los locales habitados es en muchos casos una labor no difícil de realizar.

Los aparatos, según su tipo, pueden emitir frecuencias discretas o continuas.

Entre los primeros figuran los ventiladores. Estos aparatos producen ruido como consecuencia de las compresiones y expansiones a que se ve sometido el aire por los álabes del ventilador. Su frecuencia depende del número de aletas y de la velocidad del ventilador, $\mathrm{y}$, en general, no solamente producen un sonido fundamental, sino una serie de armónicos. Su intensidad depende del tamaño y características constructivas del ventilador. 
Al sonido producido por los álabes puede unirse el producido por cojinetes en estado defectuoso, y por el motor si no está completamente equilibrado. La intensidad de estos ruidos viene ampliada por la carcasa del ventilador.

No se puede hablar de un espectro sonoro característico de los ventiladores, pues, como se ha dicho antes, depende de varios factores, $y$, por tanto, cada tipo de ventilador tiene su propio espectro para cada velocidad. En general, la mayor intensidad sonora de estos aparatos corresponde a bajas frecuencias o frecuencias intermedias dentro del campo audible. Un espectro propio de un ventilador puede ser el indicado en la figura 2.

El ruido interno de los ventiladores tiene distintas características que el externo, y la forma de transmitirse uno $y$ otro a los locales habitados es también diferente. El ruido interno, es decir, el que apreciaría un espectador o un micrófono situado en el interior del conducto de salida, es transmitido a los locales acondicionados principalmente a través del aire impulsado. El ruido externo, es decir, el apreciado por un espectador situado junto al ventilador, es transmitido a las habitaciones a través de la estructura y el espacio de las edificaciones. La manera de evitar la propagación de uno y otro es, naturalmente, distinta, y de ello haremos mención más adelante.

Los conductos también pueden generar sonidos de frecuencias discretas. Los ruidos producidos en ellos pueden ser debidos a:

- Los torbellinos de aire creados por obstáculos interpuestos en los conductos.

- La resonancia de las paredes de estos conductos.

- La transmisión a los conductos de ruidos procedentes de la edificación.

La última causa depende únicamente del equipo y del montaje, mientras que las otras dos dependen también de las características de funcionamiento y, concretamente, de la velocidad del aire en los conductos.

Su evaluación «a priori» no es fácil ni cualitativa ni cuantitativamente, aunque existen datos que permiten una cierta aproximación. En este campo también se están llevando a cabo investigaciones para dar mayores aproximaciones a las características sonoras del ruido originado en estos casos. Su importancia puede ser considerable, especialmente en acondicionamiento de aire a alta velocidad, porque, por ejemplo, los ruidos aerodinámicos aumentan en intensidad con la quinta potencia de la velocidad y, en algunos casos especiales, hasta con la octava potencia. Las frecuencias dependen de la velocidad, de la forma del conducto y de los obstáculos en él interpuestos.

Los ruidos transmitidos por la estructura al conducto requieren un aislamiento acústico entre ambos, aislamiento que, en general, no es difícil de conseguir.

Finalmente existen una serie de partes de movimiento periódico en una instalación de aire acondicionado, todas ellas generadores en potencia de ruidos de frecuencias discretas, tales como moto- 
res eléctricos mal equilibrados magnética y mecánicamente, efectos pulsatorios de quemadores que pueden tener resonancias en el equipo de combustión, bombas, etc., que deben ser tenidos en cuenta al atender a la parte acústica del proyecto. Estos ruidos, según la situación del foco emisor, pueden ser transmitidos a los locales a través del aire, de la estructura o de ambos a la vez.

Entre las fuentes de ruidos de frecuencias continuas, podemos considerar las rejillas y demás elementos distribuidores de aire en las habitaciones.

Estos elementos deben proyectarse y elegirse con el máximo cuidado, pues sus ruidos son transmitidos íntegramente al local, ya que, por estar situados en la misma habitación, no hay forma de colocar un absorbente acústico que evite su propagación a la misma. Si a esto se añade que cuando estos elementos producen ruido la intensidad de éste es mayor en frecuencias medias (teniendo valores apreciables en las bandas sonoras normales de la conversación), queda más destacada la importancia de su estudio y selección.

Las características del ruido emitido por estos distribuidores de aire a las habitaciones depende de la forma de aquéllas y de la velocidad del aire que pasa a su través. En general las intensidades sonoras en frecuencias bajas vienen afectadas, principalmente, por el volumen de aire que atraviesa la rejilla, por su tamaño y, en las rejillas regulables, por su abertura, mientras que las frecuencias altas dependen de la velocidad de paso del aire por la rejilla y de la pérdida de carga que ésta ofrece.

En la figura 3 puede apreciarse el espectro sonoro de un difusor.

El ruido producido por una rejilla o difusor crece de forma que su nivel sonoro, en decibelios, es una función proporcional al logaritmo de la velocidad. En la figura 4 se aprecia claramente esta dependencia, así como la importancia de la conformación de la rejilla o difusor.

En esta figura la curva $A$ corresponde a una rejilla cuyo chorro de aire es casi unidireccional; la curva $B$ representa una rejilla con un chorro de aire muy abierto, y la $C$ es propia de un difusor de $25 \mathrm{~cm}$ de cuello.

Cuando inmediatamente antes de una rejilla hay una válvula de regulación de caudal, ésta incrementa el sonido emitido por aquélla en una cantidad no despreciable, que depende del porcentaje de abertura. Así, por ejemplo, una válvula abierta en un 50 por 100 aumenta el nivel sonoro en aproximadamente 16 decibelios, mientras que una válvula correcta, completamente abierta, no tiene prácticamente influencia en el ruido producido por la rejilla.

Los conductos pueden ser además generadores de ruidos de espectro continuo. Al chocar una corriente de aire en régimen turbulento (en acondicionamiento de aire no se emplean velocidades que representen un régimen laminar) contra una pared rígida, se producen unos efectos de cambio de densidad que originan ruidos. Son fuentes de ruido, pues, todas las alteraciones en conductos (curvas, derivaciones, estrechamientos, etc.), $\mathrm{y}$, como todos los ruidos de origen aerodinámico, su intensidad crece fuertemente con la velocidad.

Otra fuente de ruidos de las características más diversas es la transmisión de sonidos entre habitaciones próximas a través de los conductos de aire acondicionado. Esta transmisión, a veces subestimada por el proyectista, puede, sin embargo, ser causa de molestias, cuando no de indiscreciones. Es únicamente un problema de distribución de conductos y rejillas. Cuando la longitud de conducto existente entre las acometidas a dos habitaciones es corta, es muy probable la transmisión de sonidos entre una y otra habitación. El ruido producido por la conversación es atenuado en parte por la misma habitación, y su transmisión a la contigua será tanto menos intensa cuanto más alejados estén los conversantes de la rejilla. En rejillas próximas al lugar en donde se habla, la atenuación producida por la habitación en el sonido que llega a ellas es prácticamente despreciable y, en este caso, la conversación puede ser oída sin dificultades en la habitación contigua.

Y, como se decía al hablar de fuentes de ruido de frecuencias discretas, pueden ser transmitidos a las habitaciones ocupadas ruidos de frecuencias continuas producidas en la edificación o en las propias instalaciones de aire acondicionado (compresores, equipos de combustión, bombas, tuberías, etc.), los cuales son transmitidos a través del aire de los conductos o de las propias instalaciones, y todas ellas deberán ser consideradas como elementos productores en potencia de ruido, y aisladas acústicamente en debida forma. 
c) Procedimientos más usuales de atenuación de ruido.

1. Ruido producido por la maquinaria.

La maquinaria transmite vibraciones y ruido a la estructura y al ambiente de la sala en que está ubicada.

El ruido transmitido a la estructura se reduce colocando amortiguadores y aislantes. Se utilizan frecuentemente como amortiguadores masas pesadas unidas rígidamente a la maquinaria, con lo que se disminuye la amplitud de las vibraciones que en ésta se producen.

Los aislantes consisten en elementos elásticos que absorben energía de vibración de la máquina, transmitiendo a la estructura solamente una parte de ella. El tipo y características del aislante debe ser elegido de acuerdo con la carga mecánica que ha de soportar y la clase de vibraciones que ha de absorber. Normalmente deben venir en catálogos las frecuencias y cargas recomendadas para su utilización, ya que éstas son distintas en cada tipo de aislante.

Como estos ruidos son transmitidos no solamente a través del anclaje, sino también por medio de las conducciones, entre los elementos productores de vibraciones y ruidos y los conductos y tuberías deben interponerse siempre uniones flexibles que impidan o atenúen la transmisión de estos sonidos.

El ruido transmitido al ambiente de la sala de máquinas ha de ser aislado en ella, evitando su propagación al resto de los locales habitados. Como los ruidos se atenúan con la distancia, una medida prudente es colocar la sala de máquinas tan alejada de los locales habitados como sea posible.
BANDAS DE UNA OCTAVA (Ciclos/segundo)

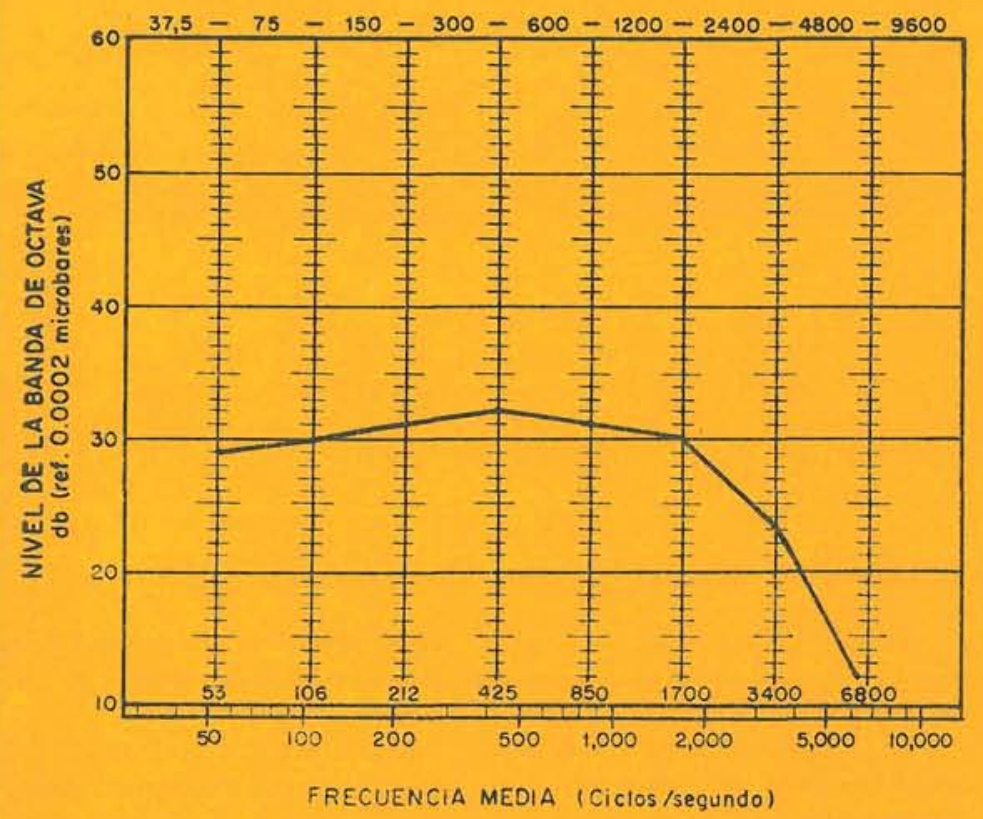

Fig. 3

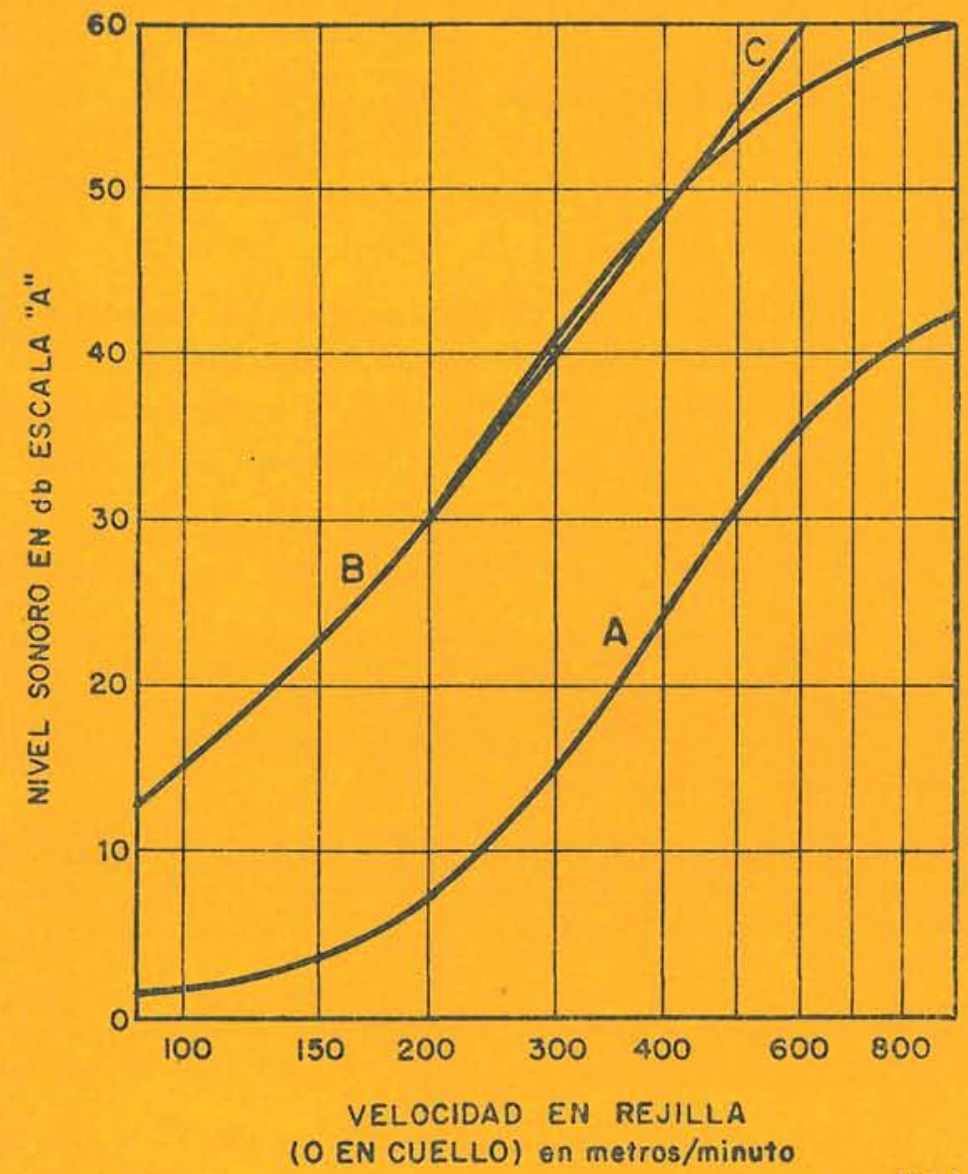

Fig. 4 


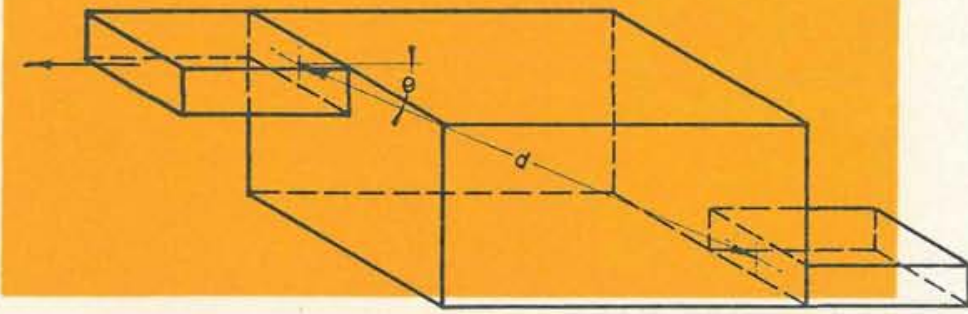

Fig. 5

Revistiendo el cuarto de máquinas de un absorbente acústico se disminuye el tiempo de reverberación en él y, por tanto, el nivel sonoro existente en este cuarto, reduciendo así el ruido de este foco emisor. La transmisión del ruido existente en el interior de la sala de máquinas al resto de la edificación se atenúa aislando acústicamente aquélla (por ejemplo, con una doble pared). Este aislamiento debe estudiarse con cuidado, pues los ruidos en la sala son en general de baja frecuencia, y es sabido que los de este tipo son los que más fácilmente se transmiten a través de las paredes.

\section{Absorción del ruido transmitido por el aire.}

El dispositivo más económico para absorber el ruido transmitido por el aire procedente del ventilador es colocar una cámara acústica después de éste.

Esta cámara produce una atenuación tanto mayor cuanto más elevado es el coeficiente de absorción acústica del material de que está recubierta interiormente, cuanto mayor es el área de la cámara, cuanto menor es el área del conducto de salida de la misma y cuanto más distantes están las bocas de entrada y salida de aire, las cuales es conveniente que no estén alineadas.

La atenuación lograda con una cámara como la dibujada en la figura 5 viene dada por:

Atenuación en decibelios $=10 \log \frac{1}{\left(\begin{array}{cc}\frac{\cos \theta}{2 \pi d^{2}} & \frac{1-\alpha}{\alpha S_{c}}\end{array}\right) S_{s}}$

En la que:

$\alpha=$ Coeficiente de absorción acústica de] aislamiento interior.

$\mathrm{S}_{c}=$ Superficie de la cámara, en metros cuadrados.

$\mathrm{S}_{s}=$ Sección del conducto de salida, en metros cuadrados.

$\theta$ y $d=$ Los valores indicados en la figura 5 ( $d$ en metros).

Los conductos que, como hemos visto antes, pueden ser generadores de ruido, en especial con aire a altas velocidades, son también absorbentes, tanto en tramos rectos como en curvas.

En tramos rectos de conductos rectangulares sin tratar acústicamente, la absorción es debida principalmente a la energía del aire que es transmitida a las paredes de los mismos. Estas paredes pueden entrar en resonancia y transmiten a su vez el ruido al ambiente exterior a las mismas, por lo que estos conductos sin tratar deben estar situados únicamente en lugares en los que no sea nocivo el sonido emitido por ellos.

La atenuación del sonido en conductos sin tratar acústicamente es variable según el tamaño y la forma de los mismos, pudiendo variar entre 0,6 y 0,2 decibelios por metro lineal de conducto, siendo mayor la atenuación de sonidos de bajas frecuencias.

Cuando los conductos pasen a través de zonas en las que no sea deseable esta emisión de sonido, pueden tratarse exteriormente con material que atenúe esta transmisión. Este efecto atenuante se consigue, por ejemplo, con el aislamiento térmico normalmente empleado para conductos.

La atenuación del sonido en las curvas se efectúa por reflexión de las ondas sonoras al chocar contra las paredes. El efecto atenuador es, naturalmente, mayor en curvas con aletas direccionales. 
En conductos circulares, en los que las paredes no entran en vibración tan fácilmente, la atenuación del sonido es menor, alcanzando valores máximos de 0,3 decibelios por metro lineal de tubo.

Finalmente, puede ser atribuida a los conductos la fuerte atenuación que sufre el sonido al salir a las habitaciones, en donde un fenómeno de reflexión «devuelve» al conducto un porcentaje elevado del sonido transportado por el aire, porcentaje que puede alcanzar valores del $90 \%$. La atenuación obtenida en las salidas de los conductos es fuerte, en especial para ruidos de frecuencias bajas, y su efecto disminuye rápidamente conforme aumenta la frecuencia o la sección de salida del conducto.

Recubriendo las paredes interiores de los conductos con absorbentes acústicos se obtienen mayores efectos amortiguadores. La atenuación por unidad de longitud, en un conducto tratado, viene expresada con cierta aproximación por la fórmula:

Atenuación en decibelios $=28,3 \frac{P}{A} \alpha^{1,4}$, por metro, siendo:

$P=$ Perímetro del conducto, en metros.

$A=$ Sección recta del conducto, en metros cuadrados.

$\alpha=$ Coeficiente de absorción del material de recubrimiento.

La atenuación depende también del espesor de la capa de absorbente colocada, siendo esta influencia notable, sobre todo para bajas frecuencias.

Es muy eficaz la absorción obtenida en las curvas tratadas acústicamente en su interior. En este caso, el absorbente eficaz es el colocado en los laterales del conducto, y su mayor efecto se logra para los sonidos cuya longitud de onda es inferior al ancho del conducto. En general, la absorción es mejor para frecuencias altas que para frecuencias bajas. Se obtienen muy buenos resultados tratando los conductos antes y después de las curvas, en una longitud al menos igual a dos veces el ancho del conducto.

Existen en el mercado diversos tipos de atenuadores de sonido prefabricados para su inserción en los conductos. Estos tipos responden a diversos modelos, entre los cuales son corrientes aquellos en los que se divide el conducto principal en una serie de conductos longitudinales por medio de tabiques tratados acústicamente, con objeto de aumentar la superficie de absorción de ruido. Otros modelos están constituidos por conductos en los que sus paredes perforadas se hallan en comuni. cación con una serie de cámaras exteriores estancas y tratadas interiormente con absorbentes acústicos.

El espectro de absorción de cada tipo y modelo es distinto, y sus características deben venir indicadas en catálogo. En general, el poder absorbente depende de la longitud; y las frecuencias absorbidas, de la forma del silenciador.

Dos son los lugares más indicados para colocar los tratamientos destinados a la atenuación del sonido: uno de ellos es en el conducto general de alimentación del aire, reduciendo así, con un solo tratamiento, el ruido procedente del ventilador y de la sala de maquinaria; está indicado otro tratamiento acústico un poco antes de las rejillas o del conducto que alimenta a varias rejillas de una habitación. Con este último tratamiento se elimina ruido de la maquinaria que no haya sido absorbido en el atenuador general, y el producido en los conductos, ajustando los valores de acuerdo con lo requerido para cada habitación.

El ruido originado en las rejillas-como se dijo anteriormente-es difícil de eliminar. Sus efectos pueden ser disminuidos mediante un tratamiento acústico de la habitación, y su colocación también puede afectar a la repartición de sonido dentro de la sala. En general, cuanto más encauzadas estén las ondas sonoras procedentes de las rejillas, tanto más pueden ser apreciadas por el observador situado en el campo barrido por las mismas. En este sentido, las rejillas menos recomendables son las situadas en los rincones de las habitaciones, y las que mejores resultados dan son las colocadas en el centro de los techos. 


\section{Gomprobacución \\ de In calledual somora de un ca instralación}



El método más práctico para comprobar la calidad sonora de una instalación, consiste en medir directamente el ruido que ésta produce

Fig. 6 en los locales habitados. Los niveles de ruido correspondientes a la escala A pueden medirse por medio de un medidor acústico comercial, preparado para efectuar lecturas en esta escala (fig. 6).

La lectura se debe hacer en varios puntos de la habitación, localizados en las zonas en donde vayan a estar los ocupantes, con lo cual la medición nos dará una idea del confort que éstos van a tener.

La apreciación del nivel sonoro de la instalación de acondicionamiento de aire se puede realizar directamente cuando sea posible eliminar de la habitación ruidos ajenos a aquélla. En el caso de

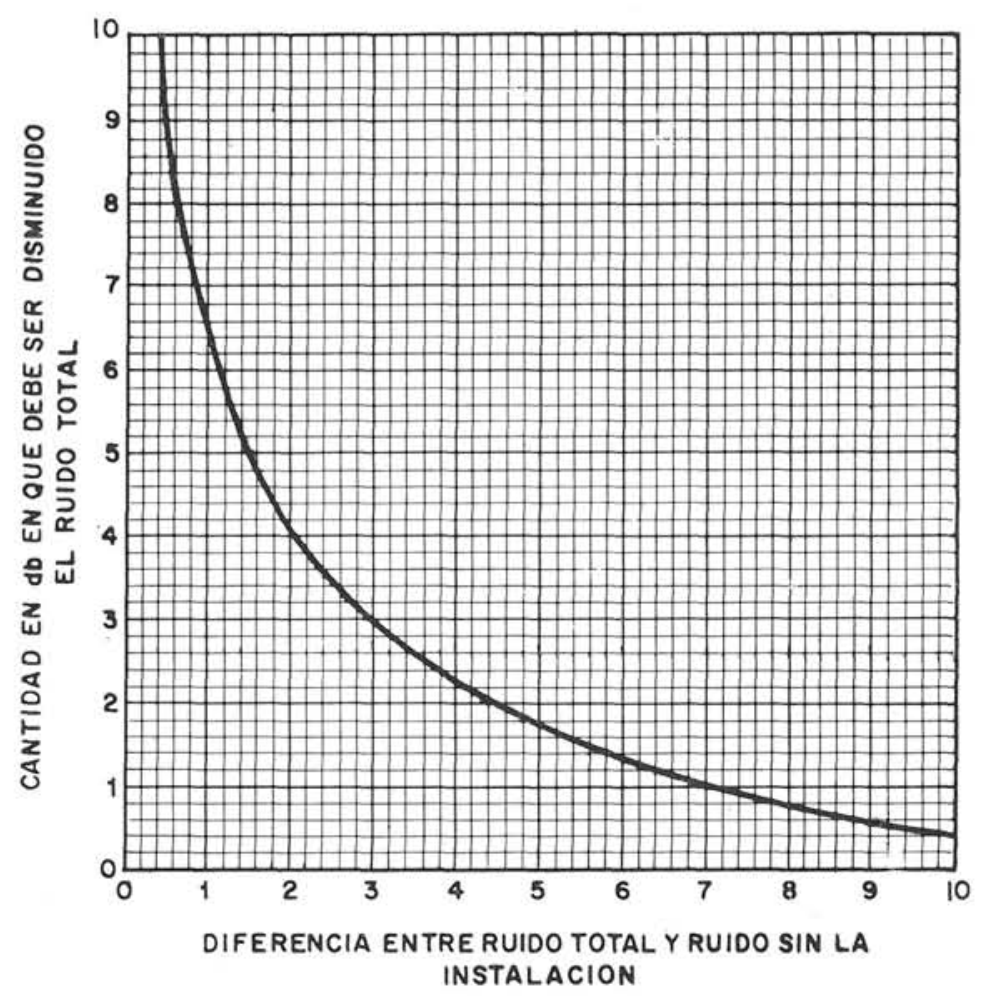

Fig. 7 que esto no pueda realizarse, la apreciación del ruido de la instalación puede realizarse por corrección del ruido total, teniendo en cuenta la diferencia entre el ruido observado cuando funciona la instalación, y el observado sin que ésta funcione. Se indica, en la figura $7,1 a$ curva de correcciones que hay que utilizar en estos casos.

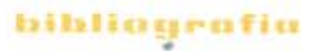

American Society of Heating ReFRIGERATING AND AIR CONDITIONING ENGINEERS: Guide 1963.

hiarris: Handbook of Noise Control, 1957.

I. E. T. c. c.: Normas Acústicas en la Edificación, 1961.

Toвío: El aislamiento y acondicionamiento acústicos en la edificación, 1960. 


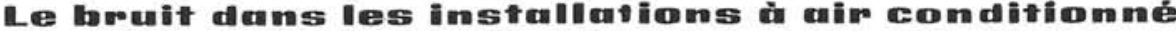

A. Alamán, Ing. industriel. Chef de la Division d'Installations de l'IETcc.

Cet article donne un aperçu général du problème du bruit dans les installations d'air conditionné, problème qui suscite de sérieuses préoccupations dans les milieux techniques correspondants pour sa grande influence sur le confort dans les édifices.

Il donne les chiffres de niveaux de pression sonore admissibles, recommandables pour l'élaboration d'un Cahier des Charges et le procédé pour vérifier si les installations terminées l'observent, ainsi que les moyens normalement utilisés pour éviter l'émission de bruit dans les locaux d'habitation.

Pour l'étude du confort sonore, il recommande de tenir compte, tant pour le Cahier des Charges (grâce à l'emploi de l'échelle «A») que pour l'étude de l'élimination de bruits lors de l'élaboration du projet de l'installation (au moyen de courbes spectrales indiquées), non seulement du niveau sonore global, mais aussi, conformément à la tendance mondiale, du spectre de fréquences de chaque niveau sonore, grâce auxquels il est possible d'arriver à un même confort, avec une installation plus économique. Pour cela, il sera très utile aux projeteurs de pouvoir compter sur la coopération des fabricants qui leur procureront les données concernant les propriétés acoustiques de leurs produits, tant de ceux pouvant émettre des bruits, que de ceux cernant les propriétés acoustiques de leurs prod
qui seront utilisés comme absorbants ou isolants.



A. Alamán, Industrial engineer. Chief of Installations Division, I. E. T. c. c.

This article gives a general account of the noise problem in air conditioning systems. This problem causes grave concern to the technicians owing to its influence on the comfort of the buildings concerned.

The acceptable levels of noise are discussed, also their codification into general specifications. Methods are given to test whether a given installation satisfies such noise specifications, and the means that are usually adopted to prevent air conditioning noise from extending into inhabited rooms.

The study of the limitation of noise to a comfortable level depends both on the noise specifications and the actual design of the installation. Scale "A» is utilised in connection with the specifications, and the sound spectral curves are part of the design technique, which enables to obtain the cheapest design for the same level of comfort. The designer needs the fullest cooperation of the manufacturers, who should supply him with detailed accoustical data about the equipment. This applies both to installations that will produce noise with detailed accoustical data about the equiph

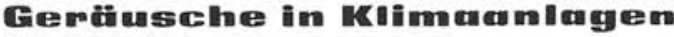

A. Alamán, Ingenieur. Leiter der Installationsabteilung des I. E. T. c. c.

Dieser Artikel behandelt ganz allgemein das Problem der Geräusche in Klimaanlagen, das wegen seines grossen Einflusses auf den Komfort eines Gebäudes die Techniker ausserordentlich beschäftigt.

Es werden Zahlen für zulässige Schallpegel angegeben, die für die technischen Bedingungen eines Projektes zu empfehlen sind und ausserdem wird ein Verfahren genannt, mit dem man prüfen kann, ob die Anlagen den Vorschriften entsprechen.

Ferner werden die üblichen Massnahmen und Mittel genannt, die angewandt werden um die Aussendung von Geräuschen in die bewohnten Räume zu verhindern.

Zum Studium des Schallkomforts empfiehlt es sich sowohl in den technischen Vorschriften (durch Anwendung der Skala «A») als auch in der Untersuchung zur Beseitigung von Geräuschen beim Entwurf einer Anlage (durch die angegebenen Spektralkurven) nicht nur den Gesamtschallogel, sondern im Einklang mit der allgemein üblichen Tendenz, das Frequenzspektrum jedes Schallpegels zu berücksichtigen, wodurch es möglich ist, mein üblichen Tendenz, das Frequenzspektrum jedes Schallpegels zu berücksichtigen, wodurch es möglich ist,
das gleiche Resultat mit einer billigeren Anlage zu erzielen. Von grosser Hilfe für den Planer wäre es dann, mit der notwendigen Zusammenarbeit der Fabrikanten rechnen zu können, indem diese Angaben über akustische Eigenschaften ihrer Produkte zur Verfügung stellen und zwar sowohl über geräuschaussendende Geräte als auch solche, die als Aufnahmegeräte oder Isolatoren verwendet werden. 